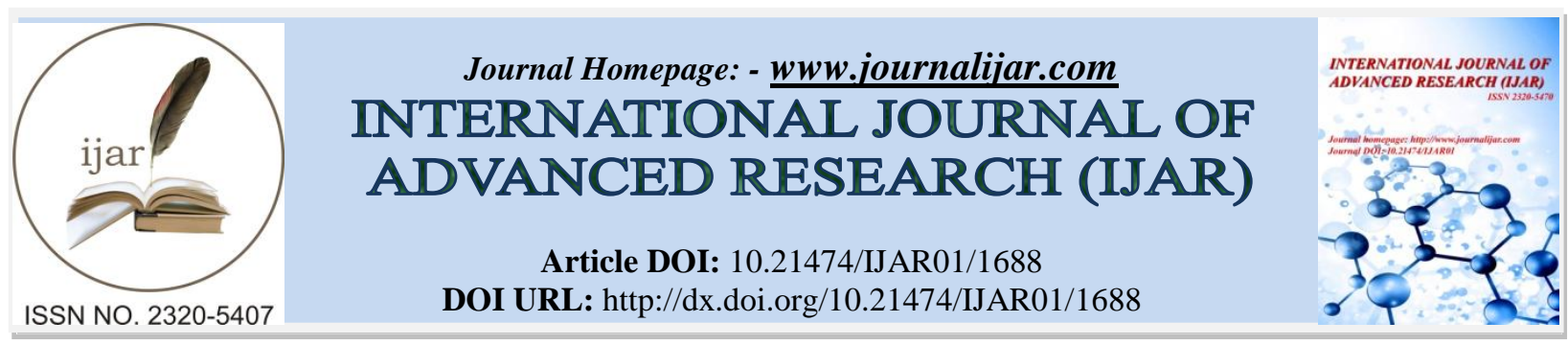

RESEARCH ARTICLE

\title{
TURN AROUND TIME A QUALITY ATTRIBUTE FOR LABORATORY PROFICIENCY : A ONE YEAR EXPERIENCE.
}

Saswati Das and B. C. Koner.

Department of Biochemistry, Maulana Azad Medical College, New Delhi-110002

\section{Manuscript Info}

\section{Manuscript History}

Received: 12 July 2016

Final Accepted: 12 August 2016

Published: September 2016

\section{Key words:-}

Turn around time, Timeliness, Quality attribute, Analytical, Preanalytical, Postanalytical

\section{Abstract}

Timeliness is an important quality attribute for any laboratory. Timeliness which is commonly expressed as the turnaround time (TAT) is often used as the benchmark for laboratory performance. Our study evaluated the total TAT, the intralab TAT and contribution of the preanalytical, analytical and post analytical phase to the total TAT. The turn around time (TAT) for routine and emergency for both the outpatients and inpatients were evaluated for one year. The total TAT was calculated from sample reception till the dispatch. The TAT for the routine biochemistry samples for inpatients was an average of $5 \mathrm{~h}$ whereas the TAT for the outpatient samples was $24 \mathrm{~h}$. The turnaround time for emergency samples was $1 \mathrm{~h}$. The contribution of the pre and post-analytical phases were $80 \%$ in OPD and $62.5 \%$ in Ward (IPD) to the total TAT whereas for emergency samples the pre and post analytical phases contribute to $55 \% \& 50 \%$ of total TAT for routine parameters and electrolytes respectively. The analysis of TAT demonstrates the need for improvement and improvisation in the preanalytical and post-analytical phase in order to reduce the total TAT.

Copy Right, IJAR, 2016,. All rights reserved.

\section{Introduction:-}

Timeliness which is commonly expressed as the turnaround time (TAT) is often used as the benchmark for laboratory performance. ${ }^{1}$ Clinicians desire a rapid, reliable and efficient service delivered at low cost. Amongst these quality attributes, timeliness is perhaps the most important to the clinician. ${ }^{2}$ Delayed TATs increase the frequency of duplicate samples sent to the laboratory which eventually increases the workload on the laboratory. It is essential to assess and improve the turnaround time to ensure proficient laboratory quality management as well as ensuring patient satisfaction. ${ }^{3}$ Turnaround time (TAT) has been described in different manner by several researchers. The College of American Pathology defines TAT as "the period of time from test ordering to the time the results are made to the emergency department". ${ }^{2}$ The term "total testing cycle" describes TAT as a combination of nine steps namely ordering, collection, identification, transport, preparation, analysis, reporting, interpretation and action. ${ }^{4}$ Clinicians consider TAT from the time the test is ordered to results reporting as TAT. However, laboratory professionals usually use specimen receipt to reporting of results as TAT. ${ }^{5}$ Another term therapeutic TAT describes the interval when a test is requested to the time some therapeutic decision is taken. ${ }^{6}$ TAT has also been classified as pre- analytical, analytical and post-analytical based on the different phases of sample processing . ${ }^{7}$ The aim of the present study is to determine the current turnaround time (TAT) of the biochemistry laboratory for the emergency 
department, in patient and out patient services The study also evaluates the contribution of analytical, pre-analytical and post-analytical phases to the total turnaround time (TAT). The steps necessary to reduce the total turnaround time were also evaluated.

\section{Material \& Methods:-}

The present study has been conducted on samples received in the biochemistry laboratory of a Lok Nayak Hospital, which is a tertiary care hospital catering to a large population of Delhi. The Clinical Biochemistry Lab of Lok Nayak Hospital is equipped with latest instruments like DXC 800 (Beckman Coulter, USA), Olympus AU 400 \& Olympus AU 480 (Beckman Coulter, USA). Our lab also has three automated Direct ISE Ecolyte analyzer by Cobas.

The samples from outdoor patients are collected at a centralized collection centre by trained phlebotomists whereas inpatient samples are drawn by the respective clinician department staff. The routine and emergency (stat) samples are both delivered to the lab by paramedical and other ancillary staff from the ward and the OPDs respectively. Quality Control (QC) samples were run daily in the laboratory twice a day for all the analytes to identify any intraassay fluctuations. The sample run is commenced only if the QC results are satisfactory. The routine ward samples are received by our technical staff in the morning and are screened for any pre-analytical errors followed by their processing. In our laboratory we have a dedicated section for processing stat samples from the Emergency Unit equipped with an autoanalyser, \& Ecolyte machines for measurement of serum electrolytes.

The laboratory support staff makes entry regarding the time of sample reception by the lab. The samples are processed in the order in which they are received for the ward samples. After the validation is complete by the clinical biochemist the reports are dispatched to the respective ward via a manual courier and the time is noted on the dispatch register.

In this paper we are presenting TAT of routine, emergency and OPD samples received by our laboratory from December 2014 to November 2015.

In our laboratory we process around 400 samples from ward(in-patient), 450 samples from OPD daily and 700 sample from the ER which are processed round the clock. The OPD samples arrive in two batches, one batch in the forenoon and other in the afternoon. The steps in processing of the samples are divided into the pre-analytical, analytical, post-analytical phase.

Pre-analytical phase (T1):

- Phlebotomist or nursing staff calls the patient and processes the request for the required investigation

- Collection of the blood sample

- Filling up of the investigation form and labelling of the samples.

- Transport of the samples from the sample collection area to the laboratory

Analytical phase (T2):

- Prerun processing of the samples: Centrifugation for serum separation.

- Samples run in the autoanalyser and report generation by the analyser.

Post-analytical phase (T3):

- Entry of the reports in the requisition form and the record register.

- Validation of the report by the biochemist.

- Dispatch of the report to the clinician via manual courier.

Intralaboratory TAT is calculated from the time the sample is received in the laboratory to the time the reports are sent for dispatch. The time required all the three phases of processing and intralab TAT for ward, OPD and emergency samples has documented in a logbook for a year. 


\section{Results:-}

The details of the average turnaround times observed in our laboratory for different parameters for a period of one year and the percentage contribution of different phases of analysis to the total TAT is documented in the following Table.

In the results compiled all the outliers were excluded. The outliers were due to the prolonged TAT which happened when there was machine breakdown and other unprecedented events like interrupted water supply, no electricity, lack of manpower.

The average total TAT for emergency samples is 1.5 to $2 \mathrm{hr}$ and for ward (in-patient) sample is $5.5 \mathrm{hr}$. The intralaboratory TAT is far less than the total TAT in both cases. Samples from the emergency unit are run on stat mode in the autoanalyser leading to an intralab TAT of $1 \mathrm{hr}$. Our results clearly indicate that the major contributing factor for prolonged TAT is the time required in the pre-analytical and post analytical phase. The average TAT for OPD samples is $24 \mathrm{hrs}$ since the reports are dispatched next day and the patient collects them on their next OPD visit.

Table 1 gives details of average turnaround times observed in our laboratory for different chemistries for a period of 1 year and Table 2: Percentage contribution of different phases of analysis to the total TAT.

Table 1:

\begin{tabular}{|l|l|l|l|}
\hline \multirow{2}{*}{ Emergency(ED) } & Parameters & Total TAT & Intralab TAT \\
\hline \multirow{2}{*}{ Ward(IPD) } & Routine Parameters & $2 \mathrm{hr}$ & $1 \mathrm{hrs}$ \\
\cline { 2 - 4 } & Electrolytes & $1 \mathrm{hrs}$ & $30 \mathrm{~min}$ \\
\hline OPD & Electrolytes & $5.0 \mathrm{hr}$ & $2 \mathrm{hr}$ \\
\cline { 2 - 4 } & Routine Chemistries & $5.5 \mathrm{hr}$ & $2 \mathrm{hr}$ \\
\hline
\end{tabular}

Table 2:

\begin{tabular}{|l|l|l|l|l|}
\hline & Parameters & $\% \mathrm{~T} 1$ & $\% \mathrm{~T} 2$ & $\% \mathrm{~T} 3$ \\
\hline \multirow{2}{*}{ Emergency(ED) } & Routine Parameters & 30 & 45 & 25 \\
\cline { 2 - 5 } & Electrolytes & 25 & 50 & 25 \\
\hline \multirow{2}{*}{ Ward(IPD) } & Electrolytes & 30 & 40 & 30 \\
\cline { 2 - 5 } & Routine Chemistries & 25 & 35 & 40 \\
\hline OPD & Routine parameters & 25 & 20 & 65 \\
\hline
\end{tabular}

\section{Discussion:-}

One of the most discussed areas of laboratory service quality is why the laboratory professionals not make timeliness of results reporting a major priority. Well the answer to that is probably that improving TAT is a difficult task. However there are many factors which contribute to the total TAT which may not be under the jurisdiction of laboratory professionals. The results of our study demonstrates that the pre and post-analytical phases are contributing $80 \%$ in OPD and $62.5 \%$ in Ward (In patient) to the total TAT whereas for emergency samples the pre (T1) and post analytical (T3) phases contribute to 55\% \& 50\% of total TAT for routine parameters and electrolytes respectively. These results indicate that if the time consumed for pre and post-analytical phases is reduced, than the total TAT can also be reduced unlike the current scenario where maximum thrust is on speeding up the analytical phase. Different methods may be adopted in each of the phases - pre-analytical, analytical and post-analytical phases in order to achieve optimum TAT. In order to minimize the delays in pre-analytical phase certain measures like adoption of ideal phlebotomy practices, use of plasma and serum separator tubes ,bar-coding of samples, use of computer generated requisition slips, will reduce the delays occurring as a result of illegible slips and wrong sample collection techniques. Inclusion of pneumatic tubing mechanism can reduce the time taken to transport the specimen from phlebotomy section to the laboratory . The pneumatic system is an innovation that has revolutionized sample transport and several studies have proven the efficiency of this system in reducing the unnecessary delays as a result of manual courier system. ${ }^{8-11}$ In a study done by Berry DE ${ }^{12}$ it was found that adoption of pneumatic tubing system led to a significant reduction of TAT. The analytical phase can be reduced by adoption of efficient quality control procedures, use of plasma or whole blood samples, proper training of technical staff to handle urgent samples with priority, using fully automated machines with higher throughput, automatic dilutions when results are above linearity and prompt validation of reports once the tests are completed. The post-analytical phase can be 
reduced by adoption of LIS (Laboratory Information System). The use of manual courier for dispatch of reports to the respective wards should cease. Instead LIS needs to be implemented so that the clinicians and staff nurses should be able to see the report on the computer and take prints. The need of the hour is also to develop a line of communication among the clinician and the laboratory professionals. A step towards this would be short history mentioned on the requisition slips by clinicians. The laboratory professionals should ensure prompt information of critical values to the respective wards and processing of repeat sample if needed. The total TAT of our laboratory can be improved further by the inclusion of pneumatic system and implementation of Laboratory Information System (LIS).

\section{Conclusion:-}

Timeliness is an important quality attribute for any laboratory. The results of our present study tell us that TAT of our laboratory can be improved further. Implementation of LIS, issue of printed reports, bar-coding of samples, sample collection by well trained phlebotomists and sample processing by competent technicians would reduce the TAT considerably. The biggest hindrance for swift TAT in our set up is dependence on a manual courier for sample transport and dispatch of reports. We as clinical biochemists need to formulate an empirical approach to obtain optimum TAT in our laboratories.

\section{Reference:-}

1. Kenagy JW, Berwick DM, Shone MF. Service quality in health care. JAMA. 1999 ; 281: 661-5.

2. Howanitz JH, Howanitz PJ. Laboratory results. Timeliness as a quality attribute and strategy. Am J Clin Pathol 2001;116:311-5.

3. Goswami B, Singh B, Chawla R, Gupta VK, Mallika V. Turn Around Time (TAT) as a Benchmark of Laboratory Performance. Indian Journal of Clinical Biochemistry. 2010;25(4):376-379. doi:10.1007/s12291010-0056-4.

4. Lundberg GD. Acting on significant laboratory results. JAMA. 1981; 245: 1762-1763. doi: 10.1001/ jama.245.21.2212.

5. Weinstein S. Quality in pathology laboratory practice. J Qual Clin Pract. 1995 ;15: 121-26.

6. Fermann GJ, Suyama J. Point of care testing in the emergency department. J Emerg Med. 2002;22:393-404.

7. Truchand A, Le Neel T, Brochard H, Malvaux S, Moyon M, Cazaubiel M. New tools for laboratory design and management. Clin Chem 1997 ; 43: 1709-15.

8. Hawkins RC. Laboratory turnaround time. Clin Biochem Rev. 2007;28(4):179-94.

9. Howanitz PJ. Errors in laboratory medicine: practical lessons to improve patient safety. Arch Pathol Lab Med. 2005;129:1252-61.

10. McQueen MJ. Role of the laboratory in meeting the needs of critical care. Clin Biochem. $1992 ; 26(1): 8-10$. doi: 10.1016/00099120(93)90005-Q.

11. Fleisher M, Schwartz MK. Automated approaches to rapid-response testing. A comparative evaluation of pointof-care and centralized laboratory testing. Am J Clin Pathol. 1995 ; 104: S18-25.

12. Berry DE. Turnaround time improvement and department-wide benefits of automation in urinalysis. Clin Leadersh Manag Rev. 2006;20:E3. 